\title{
Exchange bias and inverted hysteresis in monolithic oxide films by structural gradient
}

\author{
Mohammad Saghayezhian $\odot,{ }^{1}$ Zhen Wang, ${ }^{1,2}$ Hangwen Guo, ${ }^{1}$ Rongying Jin, ${ }^{1}$ \\ Yimei Zhu, ${ }^{2}$ Jiandi Zhang, ${ }^{1}$ and E. W. Plummer ${ }^{1, *}$ \\ ${ }^{1}$ Department of Physics and Astronomy, Louisiana State University, Baton Rouge, Louisiana 70803, USA \\ ${ }^{2}$ Condensed Matter Physics and Materials Science Department, Brookhaven National Laboratory, Upton, New York 11973, USA
}

(Received 23 September 2019; published 9 December 2019)

\begin{abstract}
Interface-driven magnetic properties such as exchange bias and inverted hysteresis are highly sought after in modern functional materials, where at least two magnetically active layers are required. The ability to achieve these functionalities in a single-layer thin film (monolithic) reduces the dimensionality while enriching the magnetism. Here we uncover a previously unseen part of the phase diagram of a monolithic epitaxial thin film of $\mathrm{La}_{0.67} \mathrm{Sr}_{0.33} \mathrm{MnO}_{3}$ on $\mathrm{SrTiO}_{3}$ which exhibits inverted hysteresis, spontaneous magnetic reversal, and exchange bias due to a structural gradient in the oxygen-octahedral network. Varying the growth conditions, we have mapped the phase diagram of this material and discovered that at a specific oxygen pressure and above a critical thickness, a complex magnetic behavior appears. Atomic-scale characterization shows that this peculiar magnetism is closely linked to a continuous structural gradient that creates three distinct regions within the monolithic film, each with a different magnetism onset. Extracting oxygen-octahedral geometry by electron microscopy, we found that the Curie temperature is directly correlated with the metal-oxygen bond angle. This study illustrates the importance of octahedral geometry in shaping the physical properties of the materials.
\end{abstract}

DOI: 10.1103/PhysRevResearch.1.033160

Introduction. The structure and composition of transition metal oxides (TMOs) are two deciding factors in determining their physical properties, providing geometrical constraints on the oxygen-octahedral network that leads to a rich phase diagram with a variety of physical properties such as superconductivity, ferroelectricity, or complex magnetic ordering [1-7]. In the bulk, the structure-composition duo is often modified via tuning the growth conditions, doping, or external stimuli such as pressure [8-11]. Compared to the bulk, in epitaxial TMO heterostructures, various additional pathways are available to systematically amend this duo [12-14], such as strain doping via inert atom implantation [15], strain engineering $[16,17]$, interfacing materials with octahedralsymmetry mismatch [18-20], or structural migration via electrical gating [21,22]. Another approach in diversifying the physical properties is the creation of gradient phases in which the composition or the corner-connected oxygen-octahedral network continuously changes across the thin film. Examples are monolithic thin films of $\mathrm{LaCoO}_{3-x}, \mathrm{La}_{2 / 3} \mathrm{Sr}_{1 / 3} \mathrm{MnO}_{3-x}$, and $\mathrm{SrCoO}_{3-x}$, where large-scale oxygen vacancy migration or creation, topotactic structural phase transition [21,23,24], and spin state manipulation $[17,24,25]$ have been observed. Change related to oxygen octahedra is the one key factor that is shared between all mentioned physical phenomena.

\footnotetext{
*wplummer@phys.lsu.edu

Published by the American Physical Society under the terms of the Creative Commons Attribution 4.0 International license. Further distribution of this work must maintain attribution to the author(s) and the published article's title, journal citation, and DOI.
}

Recently, it has been shown that the interfacial structure coupling together with composition variations near the interface leads to complex magnetic interactions [26]. In fact, the collaborating effects of change in bond geometry and composition are both necessary for observing spontaneous magnetic reversal and inverted hysteresis. Therefore, it immediately becomes clear that the controlled variation of these two quantities via growth conditions, across a single-layer thin film, can create intralayer magnetic couplings. This can provide a platform to not only access unexplored area of the material's magnetic phase diagram but also quantify the role of octahedral geometry on magnetism. Realizing an atomicscale understanding of this type of monolithic thin film is remarkable for future devices where one-step growth, use of a single material, and reduced dimensionality all serve in favor of simplifying fabrication while enriching functionality.

In this paper we show that by controlled monolithic thin film growth under different growth conditions, the magnetic properties of the material undergo drastic changes, while the films retain their structural coherency. Using $\mathrm{La}_{2 / 3} \mathrm{Sr}_{1 / 3} \mathrm{MnO}_{3}$ (LSMO) as the target material, we systematically change the oxygen pressure and film thickness to manipulate the structure and magnetism. Magnetic characterization reveals a new metamagnetic phase that appears above a critical thickness, exhibiting a variety of magnetic properties such as large exchange bias, magnetization reversal, and inverted hysteresis. These properties are not a product of defects or imperfections and they are absent in bulk form, and thin films grown above or below a critical oxygen pressure. Using atomically resolved transmission electron microscopy (STEM) and electron energy loss spectroscopy (EELS) we have studied the structure and composition across the thin films. Counterintuitively, STEM and EELS analyses show 
that oxygen pressure and oxygen content in the film do not have a linear relationship, where less oxygen pressure does not necessarily create more oxygen deficiency. By measuring the metal-oxygen-metal bond length and angle for three films with different magnetic properties that were grown under different oxygen pressures, we find that the bandwidth calculated from bond length and angle [27] does not change, proving that the commonly accepted bandwidth-ferromagnetism relation is oversimplified, and not valid in these materials. Rather, the continuous gradient of the bond angle in corner-shared octahedra is responsible for intriguing magnetism. Also, we show that macroscopic structural coherence determined from $\mathrm{x}$-ray diffraction (XRD), while being a good measure of film quality, is not sufficient to probe detailed structural changes. In addition, our data suggest that change in the oxidation state of metal and oxygen is related to the structural gradient that modifies the bond hybridization, where without the atomicscale structural data, the oxidation state change is often justified within a charge transfer scenario. This study illustrates the potential of monolithic films for technological applications and exemplifies the crucial role of atomic-scale measurements for proper understanding of the underlying physics.

Magnetic characterization and phase diagram. Thin films of LSMO are grown on $\mathrm{SrTiO}_{3}$ (001) substrate (STO) under different oxygen pressures and film thicknesses using pulsed laser deposition. The thin-film growth details are presented in the Supplemental Material [28]. Magnetic properties of the thin films are measured using a superconducting quantum interference device (SQUID). Figures 1(a) and 1(b) show the magnetization as a function of magnetic field, $M(H)$, and temperature, $M(T)$, for 50 unit cell (u.c.) LSMO thin films grown under 10, 40, and 80 mTorr oxygen pressure, labeled $\mathrm{LSMO}_{10}, \mathrm{LSMO}_{40}$, and $\mathrm{LSMO}_{80}$, respectively. The magnetic hysteresis of $\mathrm{LSMO}_{80}$ and that of $\mathrm{LSMO}_{10}$ are qualitatively similar, with slightly larger magnetic saturation and smaller coercivity in $\mathrm{LSMO}_{80}$. In contrast, $\mathrm{LSMO}_{40}$ is drastically different. $\mathrm{LSMO}_{40}$ has $\sim 4$ times smaller magnetic saturation value, with more than 25 times larger coercivity, which is unexpected in a magnetic material that is known to be very soft [29]. In addition, $\mathrm{LSMO}_{40}$ shows a large exchange bias that is absent in both $\mathrm{LSMO}_{80}$ and $\mathrm{LSMO}_{10}$. Figures 1(c)-1(i) present the magnetic characteristics of $\mathrm{LSMO}_{40}$. As seen in Fig. 1(c), the magnetic hysteresis loop of $\mathrm{LSMO}_{40}$ at different temperatures is shifted to negative field, indicating the existence of negative exchange bias. The exchange bias and coercivity as a function of temperature are shown in Fig. 1(d). The negative exchange bias rapidly decreases with increasing temperature. Around $25 \mathrm{~K}$, it changes sign and becomes positive, but very small and vanishes above $40 \mathrm{~K}$. The positive exchange bias is a result of unidirectional coercivity enhancement (Fig. S3 of the Supplemental Material [28]), where the left and right coercive fields do not change in the same way [30]. The magnetization $M(T)$ for $\mathrm{LSMO}_{40}$ is shown in Fig. 1(e), where $\mathrm{LSMO}_{40}$ is cooled under zero magnetic field (ZFC) and warmed up under different magnetic fields (FW). Two features are observed, a magnetic reversal and two ferromagnetic (FM) transitions. The reversal temperature increases with decreasing magnetic field. At low temperatures, the coercivity is high, therefore requiring higher magnetic field to trigger a complete magnetization reversal.
The first FM transition (FM-1) occurs at $301 \mathrm{~K}$ and the second (FM-2) at $198 \mathrm{~K}$. Figures 1(f)-1(i) show the magnetic hysteresis measured at $T=50,100,150$, and $200 \mathrm{~K}$, respectively. As discussed earlier, $\mathrm{LSMO}_{40}$ shows exchange bias up to $40 \mathrm{~K}$. At $50 \mathrm{~K}$ the magnetic hysteresis is reminiscent of a normal FM. As temperature increases, the left and right coercive fields start to move toward each other so that they overlap at zero magnetic field at $100 \mathrm{~K}$, while still having hysteresis. At higher temperatures, the right (left) coercive field continues to move to the left (right), which is seen at temperatures 150 and $200 \mathrm{~K}$, in Figs. 1(h) and 1(i). This trend leads to the appearance of partially inverted hysteresis. The very rich magnetic phase diagram of $\mathrm{LSMO}_{40}$ is illustrated in Fig. 1(e) totally unexpected in a monolithic thin film and it is not observed in the thin films grown under other oxygen pressures.

To map the new magnetic phase diagram, we have systematically grown LSMO thin films, varying oxygen pressure and film thickness. Figure 1 shows the false-color plot of the coercivity [panel (j)] and exchange bias [panel (k)] as a function of oxygen pressure and film thickness. The white filled circles are our data points. Interestingly, we observe that above and below $P_{\mathrm{O}}=40$ mTorr, the coercivity is small with small or no exchange bias, while at $P_{\mathrm{O}}=40$ mTorr, coercivity enhancement and exchange bias only appear in films with thicknesses above 15 u.c. At 40 u.c. the exchange bias appears as high as 622 Oe. Coercivity and exchange bias maps show that both dimensionality and growth conditions are crucial in observing the complex magnetic behavior and that it is limited to a narrow growth condition, only above a critical thickness.

Atomic-scale structure and composition characteristics. LSMO has a very diverse phase diagram in terms of spin and lattice coupling, often leading to coupled magnetic and structural transitions [31]. To visualize and understand the underlying mechanism of the observed complex magnetic behavior on the atomic scale, $\mathrm{LSMO}_{10}, \mathrm{LSMO}_{40}$, and $\mathrm{LSMO}_{80}$ are studied using high-resolution transmission electron microscopy and spectroscopy. Figures 2(a)-2(c) show the structure and stoichiometry of $\mathrm{LSMO}_{80}, \mathrm{LSMO}_{40}$, and $\mathrm{LSMO}_{10}$, respectively. Careful analysis of high-angle annular dark-field (HAADF) and annular bright-field (ABF) STEM images and EELS spectra maps reveals the oxygen-octahedral tilt (OOT) angles, out-of-plane (OOP) lattice constant, and $\mathrm{La}$ and $\mathrm{Sr}$ stoichiometry across the film layer by layer.

The oxygen-octahedral network undergoes a nonmonotonic change as a function of oxygen pressure. The OOT in an LSMO film viewed along the [110] pseudocubic direction is determined from the oxygen position in $\mathrm{ABF}$ images. As shown in Figs. 2(a) and 2(b), OOT (Mn-O-Mn bond angle) in $\mathrm{LSMO}_{80}$ is the largest with $\mathrm{LSMO}_{10}$ being slightly smaller. They follow a similar behavior where OOT is suppressed near the interface and away from that it increases and remains constant, with average values of $6.1^{\circ} \pm 0.4^{\circ}$ and $5.1^{\circ} \pm 0.4^{\circ}$ for $\mathrm{LSMO}_{40}$ and $\mathrm{LSMO}_{10}$, respectively. However, the evolution of OOT in $\mathrm{LSMO}_{40}$ is remarkably different [Fig. 2(c)]. In $\mathrm{LSMO}_{40}$, the first $\sim 17$ unit cells exhibit a rise and fall of OOT. The OOT in $\mathrm{LSMO}_{40}$, suppressed at the interface (first unit cell, $4^{\circ} \pm 1^{\circ}$ ), increases to $6.2^{\circ} \pm 0.9^{\circ}$ in the fifth unit cell and then gradually decreases and finally vanishes above around the 17th unit cell. The OOT variation is accompanied 
(a)

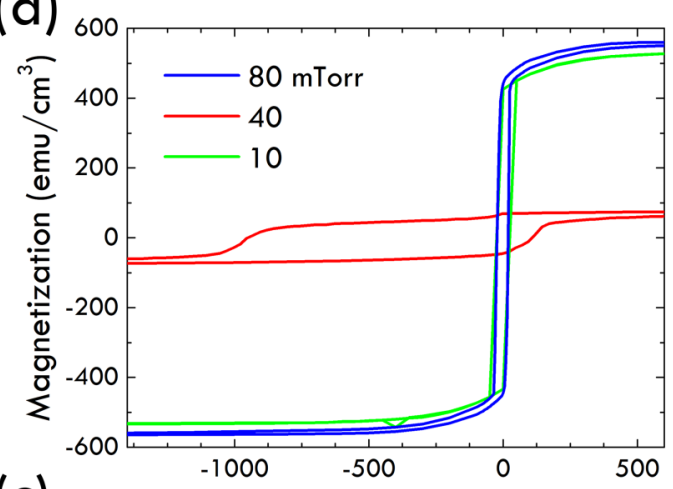

(c)

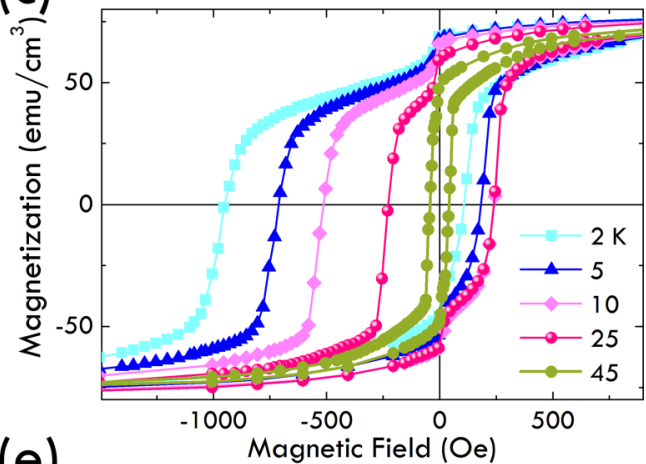

(e)

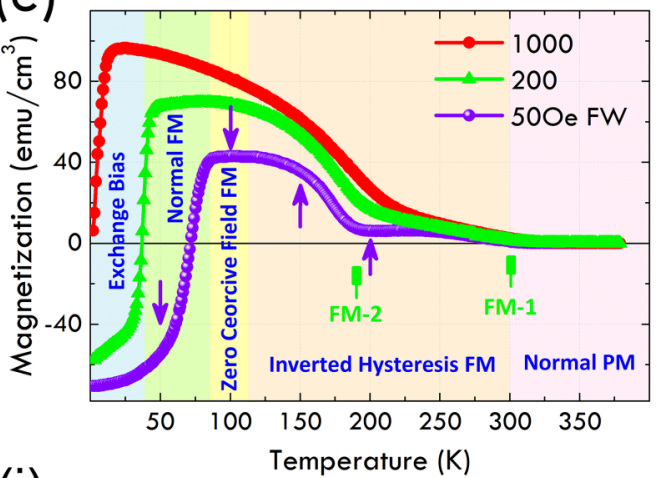

(b)

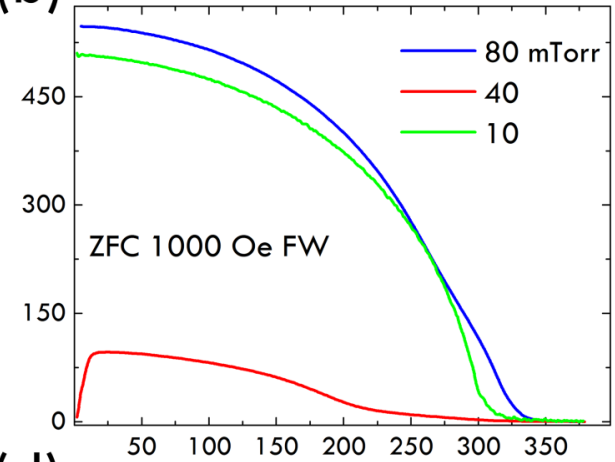

(d)
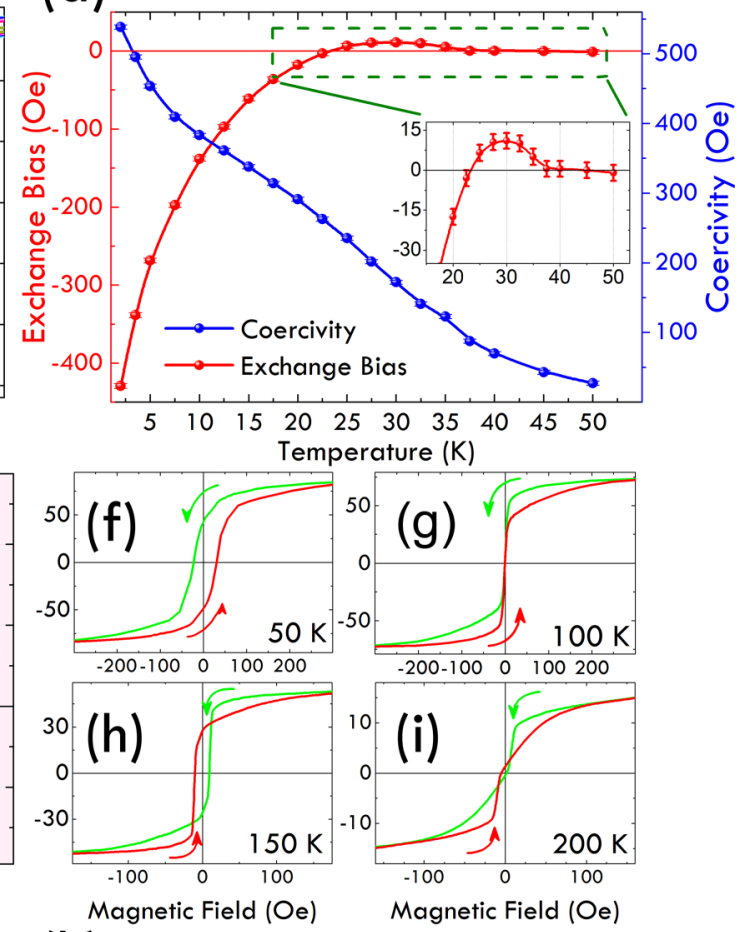

(k)
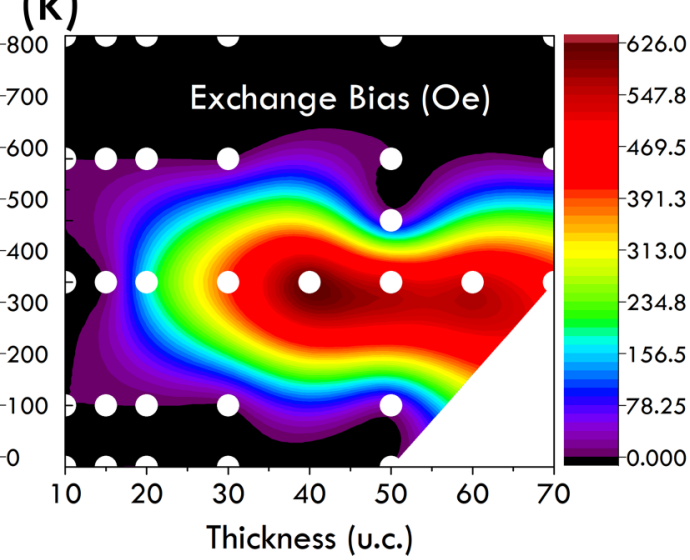

FIG. 1. Magnetic properties of the LSMO/STO (001) across the phase diagram. (a) Magnetic hysteresis of $\mathrm{LSMO}_{80}$, $\mathrm{LSMO}_{40}$, and $\mathrm{LSMO}_{10}$. While the magnetic saturation is smaller, the coercivity is much larger. (b) The temperature dependence of magnetization. (c) Temperature-dependent magnetic hysteresis for $\mathrm{LSMO}_{40}$. (d) Exchange bias and coercivity as a function of temperature for LSMO The inset shows the sign change in exchange bias. (e) Temperature dependence of magnetization for $\mathrm{LSMO}_{40}$, where it was cooled under zero field and measured under various warming fields. (f)-(i) Magnetic hysteresis at higher temperatures. Inverted hysteresis appears and develops. (j) Coercivity and (k) exchange bias as a function of film thickness and oxygen pressure. There is critical oxygen pressure (40 mTorr) and critical thickness $(15$ u.c.) for the exchange bias and inverted hysteresis. The white filled circles are the data points for thin films. The color scale is shown next to each plot. 


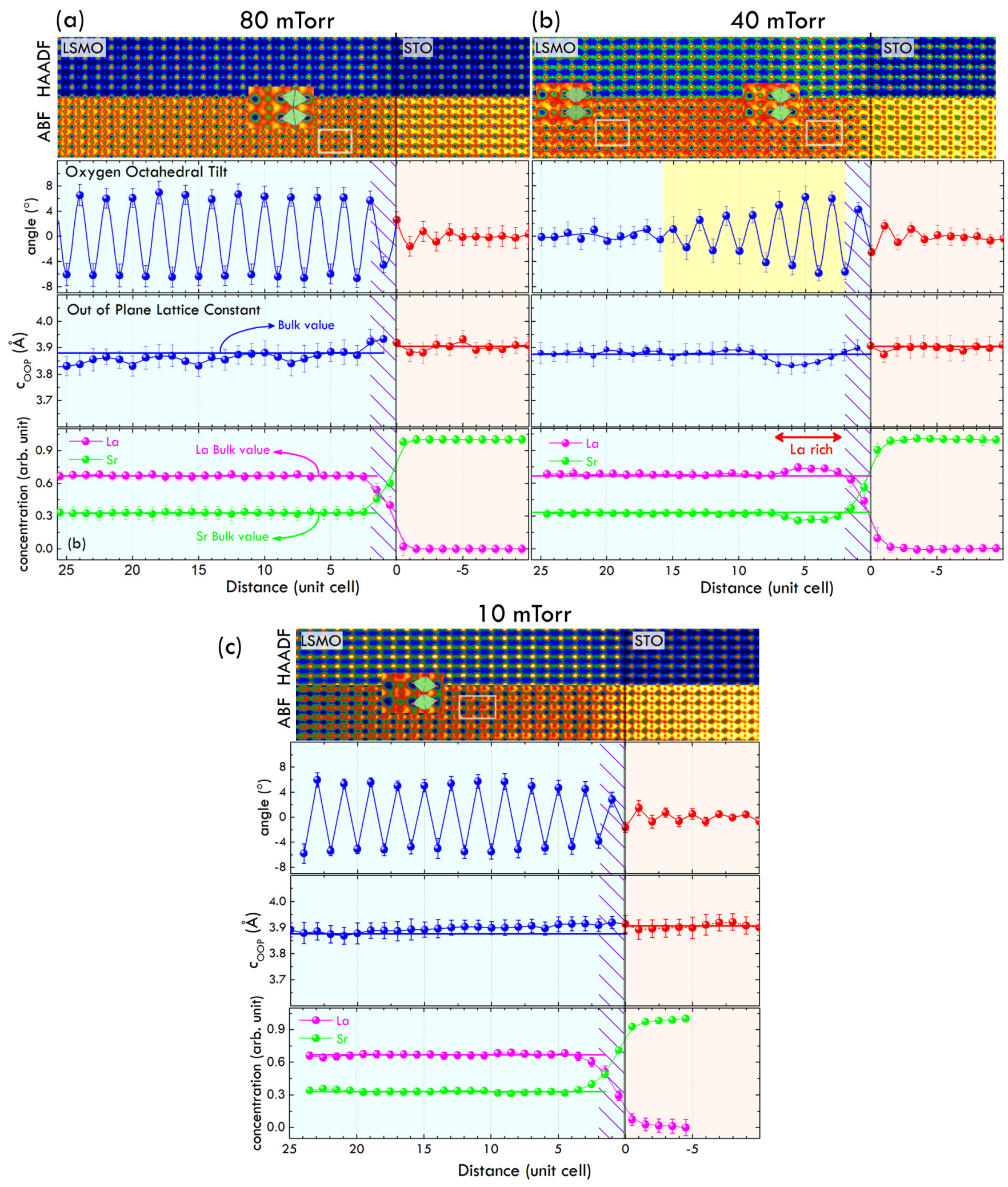

FIG. 2. The STEM microscopy and spectroscopy for (a) $\mathrm{LSMO}_{80}$ [26], (b) $\mathrm{LSMO}_{40}$, and (c) $\mathrm{LSMO}_{10}$. For each film, the HAADF and ABF images, OOT, OOP, and La and Sr concentrations are shown. The region near the interface is hatched to show the transition to the region due to substrate proximity. Except for $\mathrm{LSMO}_{40}$, above this region films recover their composition and structure. The locally modified composition structure of $\mathrm{LSMO}_{40}$ is yellow shaded, where there is a La-rich region, high OOT, and low OOP. Above this region, OOT damps to zero. 
by a change in OOP. The OOP in the first unit cell of $\mathrm{LSMO}_{40}$ is elongated (similarly to $\mathrm{LSMO}_{80}$ and $\mathrm{LSMO}_{10}$ ). From the second to the eighth unit cells, a pronounced dip in OOP is observed. Above the eighth unit cell, the OOP remains a constant. The difference between the OOP lattice constants at the two places where the tilt is maximum and the place where the tilt vanishedes controls the distance over which the OOT damping occurs; i.e., the larger the difference, the longer the damping. The OOP in $\mathrm{LSMO}_{80}$ and that in $\mathrm{LSMO}_{10}$ have a monotonic variation where the average OOP in the former (latter) is smaller (slightly larger) than the bulk, while $\mathrm{LSMO}_{40}$ is distinctly different.

Not only the local structure responds to the change in oxygen pressure, but also the stoichiometry varies as well. The $\mathrm{La}$ and $\mathrm{Sr}$ compositions for $\mathrm{LSMO}_{80}, \mathrm{LSMO}_{40}$, and $\mathrm{LSMO}_{10}$ are presented in Fig. 2. While the composition in $\mathrm{LSMO}_{40}$ is obviously distinct, $\mathrm{LSMO}_{80}$ and $\mathrm{LSMO}_{10}$ are almost the same with slight differences near the interface. $\mathrm{La}$ and $\mathrm{Sr}$ stoichiometry in $\mathrm{LSMO}_{80}$ recovers the bulk value one unit cell away from the interface, while the same occurs in $\mathrm{LSMO}_{10}$ after two unit cells. In the case of $\mathrm{LSMO}_{40}$, it returns to its $\mathrm{La}_{0.67} \mathrm{Sr}_{0.33}$ stoichiometric value after a slightly La-rich region of $\mathrm{La}_{0.72} \mathrm{Sr}_{0.28}$, from the second to the seventh unit cell.

Discussion. The emergent magnetic properties along with a gradient in structure and composition show that LSMO films carry tremendous potential for technological applications and tunability in structure-composition duo. These findings present the extraordinary functionalities emerging from a monolithic thin film (exchange bias, inverted hysteresis) and a simple route that can be expanded to other TMOs, and to other growth parameters. The change in oxygen pressure leads to a change in the plasma dynamics of the laser plume [32]. The varied oxygen pressure in $\left(A_{1-x} A^{\prime} x\right) B \mathrm{O}_{3}$ perovskites alters the $A / A^{\prime}$ or $A / B$ ratio [33]. Consequently, the change in the O-vacancy content and/or $A / A^{\prime}$ ratio changes the structure. The microscopic relations between magnetism, structure, and composition are key in finding the prime factor in determining the magnetic properties. It is often assumed that the lowered magnetization in LSMO films grown under low oxygen pressure is due to the presence of oxygen vacancies [34-37]. On the contrary, the STEM images (Fig. 2) together with magnetization data [Fig. 1(b)] show that the oxygen pressure is not correlated with the saturated magnetization in a monotonic fashion. The Mn-O-Mn bond angle drastically changes, where the smaller the angle (closer to $180^{\circ}$ ), the smaller the saturated magnetization and Curie temperature. This resembles the structural delta doping where local exchange interactions were modified through interface engineering in a multilayer thin film [18]. The advantage in the present work lies in the fact that the structural delta doping is realized in a monolithic thin film.

To explore the relation between structure and magnetic properties, we have plotted the Curie temperature as a function of average OOT in Fig. 3(a). Since $\mathrm{LSMO}_{40}$ has two FM transitions, the data points for $\mathrm{LSMO}_{40}$ in the tilted region (FM-1) and the damped region (FM-2) are shown separately. In the bulk, the OOT has the highest value $\left(6.9^{\circ}\right)$ and $T_{c}$ (370 K) [27]. In thin films, our data show that the $T_{c}$ linearly decreases with reducing the OOT, which demonstrates the intimate connection between structure and magnetism. This
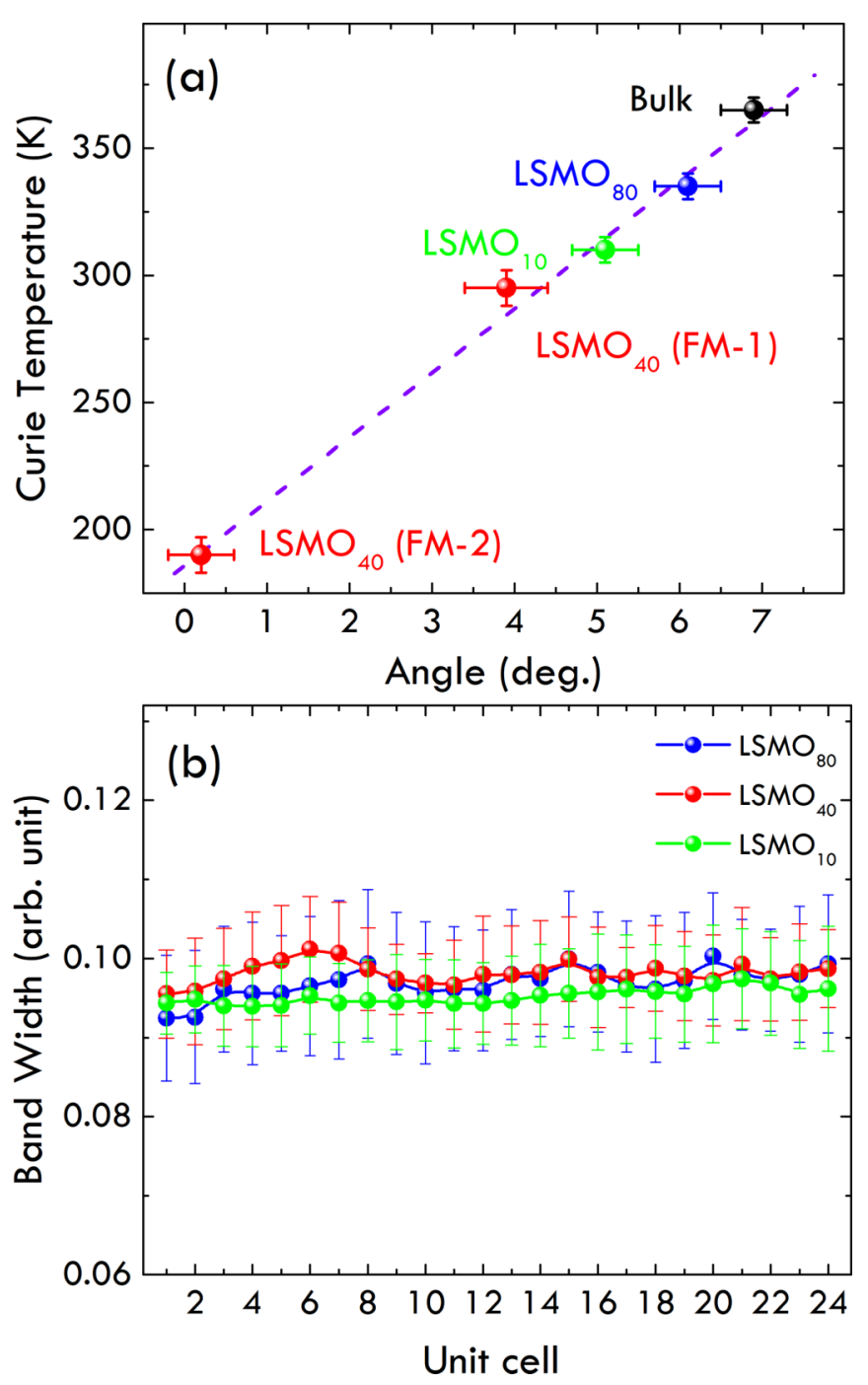

FIG. 3. (a) Curie temperature is plotted against average OOT angle; a smaller angle leads to lower $T_{c}$. (b) Bandwidth extracted from layer-resolved average $\mathrm{Mn}-\mathrm{O}-\mathrm{Mn}$ angle and $\mathrm{Mn}-\mathrm{O}$ bond length. Despite obvious differences in magnetic and electronic properties, the bandwidth remains the same.

observation illustrates that the structure, rather than oxygen pressure, plays the major role in magnetic properties.

It has been assumed that the bandwidth $(W)$ in TMOs is related to $\mathrm{Mn}-\mathrm{O}-\mathrm{Mn}$ bond angle and $\mathrm{Mn}-\mathrm{O}$ bond length. This empirical relation, $W \sim \cos \theta / d_{\mathrm{Mn}-\mathrm{O}}^{3.5}$, has been often used to justify the changes in Curie temperature [38-40]. However, the validity of this model has never been examined on the atomic scale. Using the layer-resolved bond length and bond angles, we can determine the validity of this relation on the microscopic scale. After extracting the $\mathrm{Mn}-\mathrm{O}$ bond length and angle from STEM images across the thin films, Fig. 3(b) uses this relation to show the bandwidth for $\mathrm{LSMO}_{80}, \mathrm{LSMO}_{40}$, and $\mathrm{LSMO}_{10}$, where all the films trend similarly, despite the dissimilarity in magnetic properties. The small rise in $W$ from the 2nd to the 9th unit cell in $\mathrm{LSMO}_{40}$ is due to the dip in OOP lattice constant. Above that, all samples show a similar trend indicating that the above relation is not applicable to these films. The average variation in $\mathrm{LSMO}_{40}$ compared to $\mathrm{LSMO}_{80}$ 


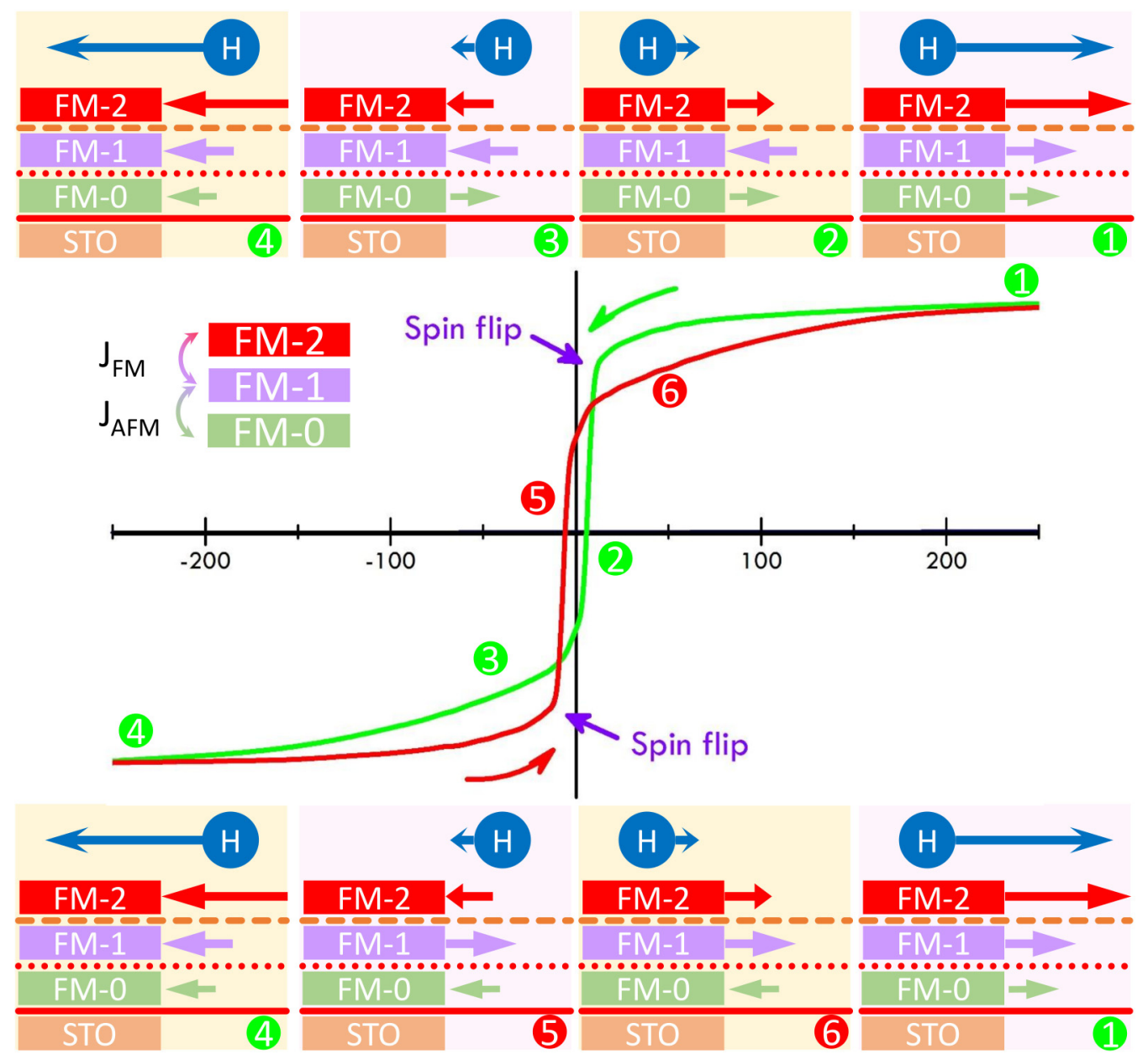

FIG. 4. Triple-layer magnetic configuration for $\mathrm{LSMO}_{40}$ and a schematic model for inverted hysteresis. The internal AFM interaction assists the magnetic reversal at low fields (spin-flip arrow) that leads to apparent inverted hysteresis.

is less than $3 \%$, while the change in $T_{c}$ is more than $43 \%$. Therefore, while bandwidth might be a contributing factor in $T_{c}$ change, it is unlikely to be the leading factor. The gradient in OOT changes the orbital hybridization of $\mathrm{O} 2 p$ and $\mathrm{Mn}$ $3 d$, which in turn changes the density of states near the Fermi energy. This is clearly observed in EELS spectra of the $\mathrm{O} K$ edge and Mn $L$ edge (Fig. S4 of the Supplemental Material [28]), which is an indication of change in unoccupied states that is related to the change in the Mn-O bond geometry. We note that the shift in energy of the $\mathrm{O} K$ edge and $\mathrm{Mn} L$ edge is often interpreted as charge transfer [41,42]. However, in light of the obvious structural changes and metallicity of these thin films, no charge transfer is required to justify the energy shifts; rather rearrangement of empty states above the Fermi energy can produce the same outcome in EELS [43]. The exact origin of the linear relation between $T_{c}$ and OOT is yet to be identified. Within the standard double-exchange model [44], lowering the OOT is expected to enhance FM as it increases electron hopping, paradoxically to our observations [45]. Our data show that the consequences of the change in OOT are not limited to electron hopping, which means a successful interpretation requires the consideration of other effects such as preferred orbital population and Jahn-Teller distortion.
We have utilized the electron microscopy and spectroscopy data to understand the peculiar magnetic properties in $\mathrm{LSMO}_{40}$. Based on the insights from STEM images, three structurally distinct regions in $\mathrm{LSMO}_{40}$ are seen (Fig. 2). They are the 1st LSMO unit cell (hatched), the enhanced OOT region (2nd to $\sim 16$ th unit cell, yellow shaded), and the damped OOT region (from the $\sim 17$ th unit cell to the surface). Comparing this to $M(T)$ in Fig. 1(e), it becomes clear that the two onsets of FM (FM-1 and FM-2) originate from enhanced and damped OOT regions, respectively. Given that $\mathrm{LSMO}_{80}$ and $\mathrm{LSMO}_{10}$ have shown that large OOT is directly related to higher Curie temperature (noting that La excess in the La-rich region is too small to create a $T_{c}$ change of $100 \mathrm{~K}$ ), it is inferred that in $\mathrm{LSMO}_{40}$ the enhanced OOT region is responsible for FM-1 and the damped OOT region for FM-2. Figure 4 shows a schematic that suggests an explanation for the hysteresis inversion based on the observed triple regions in STEM. The onsets of magnetizations show that FM-1 and FM-2 couple ferromagnetically, while at the interface, antiferromagnetic (AFM) coupling is expected where there is a considerable amount of excess $\mathrm{Sr}[26,46,47]$. At high magnetic fields in the positive direction, all moments are aligned in the direction of the magnetic field. As the magnetic field is decreased, at low magnetic fields, the AFM 
coupling prevails and FM-1 reverses (Fig. 4, step 1). FM-1 reversal slowly triggers the rotation of FM-2 (step 2). At this point, although the magnetic field is in the positive direction, the magnetization begins the reversal. As the magnetic field sweeping crosses zero, progressively more magnetic moments reverse, and total magnetization becomes stronger in the negative direction (step 3). At high negative magnetic fields, once again all moments align in the direction of the magnetic field which resets the magnetization reversal process (step 4 and onward). This type of hysteresis inversion is possible when AFM coupling is present, and the magnetic anisotropy of FM-0 is larger than that of FM-1 and FM-2 [48]. $\mathrm{LSMO}_{80}$ and $\mathrm{LSMO}_{10}$ show that larger OOT yields smaller magnetic anisotropy and hence smaller coercivity. Therefore, FM-1 that shows enhanced OOT is likely to be softer than FM-0 and FM-2, which agrees with the assumption of progressive rotation of FM-1 before FM-2.

In summary, we have shown that the monolithic TMO thin films can deliver a rich magnetic phase diagram that so far has only been accessible through heterostructuring. Our STEM data have proven to be vital in understanding the role of structure and stoichiometry in the emergent magnetic properties. They show the presence of a gradient in the structure separating the thin films into two magnetically distinct regions. In particular, it is possible to manipulate magnetic properties by modifying the oxygen-octahedra network through controlled growth conditions, rather by interfacing it with other TMOs or strain engineering. This work demonstrates a route in exploring the hidden complex magnetic phases in TMOs that are absent in their bulk counterparts.

Acknowledgments. This work is primarily supported by the US Department of Energy (DOE) under Grant No. DOE DE-SC0002136. The electronic microscopic work done at Brookhaven National Laboratory is sponsored by the US DOE Basic Energy Sciences, Materials Sciences and Engineering Division, under Contract No. DE-AC02-98CH10886. The TEM sample was prepared at the Center for Functional Nanomaterials, which is a US DOE Office of Science Facility, at Brookhaven National Laboratory under Contract No. DE-SC0012704. XRD measurements were performed at the Shared Instrumentation Facility at Louisiana State University.
[1] B. Chen, H. Xu, C. Ma, S. Mattauch, D. Lan, F. Jin, Z. Guo, S. Wan, P. Chen, G. Gao, F. Chen, Y. Su, and W. Wu, Science 357, 191 (2017).

[2] M. Gibert, P. Zubko, R. Scherwitzl, J. Íñiguez, and J.-M. Triscone, Nat. Mater. 11, 195 (2012).

[3] N. P. Armitage, P. Fournier, and R. L. Greene, Rev. Mod. Phys. 82, 2421 (2010).

[4] J. Hemberger, A. Krimmel, T. Kurz, H.-A. Krug von Nidda, V. Y. Ivanov, A. A. Mukhin, A. M. Balbashov, and A. Loidl, Phys. Rev. B 66, 094410 (2002).

[5] T. Kimura, G. Lawes, T. Goto, Y. Tokura, and A. P. Ramirez, Phys. Rev. B 71, 224425 (2005).

[6] M. Kofu, S. H. Lee, M. Fujita, H. J. Kang, H. Eisaki, and K. Yamada, Phys. Rev. Lett. 102, 047001 (2009).

[7] A. Urushibara, Y. Moritomo, T. Arima, A. Asamitsu, G. Kido, and Y. Tokura, Phys. Rev. B 51, 14103 (1995).

[8] M. L. Medarde, J. Phys.: Condens. Matter 9, 1679 (1997).

[9] Y. Tokura, Rep. Prog. Phys. 69, 797 (2006).

[10] Q. Huang, A. Santoro, J. W. Lynn, R. W. Erwin, J. A. Borchers, J. L. Peng, and R. L. Greene, Phys. Rev. B 55, 14987 (1997).

[11] N. Biškup, J. Salafranca, V. Mehta, M. P. Oxley, Y. Suzuki, S. J. Pennycook, S. T. Pantelides, and M. Varela, Phys. Rev. Lett. 112, 087202 (2014)

[12] S. Catalano, J. Kreisel, M. Gibert, J.-M. Triscone, J. Íñiguez, and J. Fowlie, Rep. Prog. Phys. 81, 046501 (2017).

[13] A. Bhattacharya and S. J. May, Annu. Rev. Mater. Res. 44, 65 (2014).

[14] J. M. Rondinelli and N. A. Spaldin, Adv. Mater. 23, 3363 (2011).

[15] H. Guo, S. Dong, P. D. Rack, J. D. Budai, C. Beekman, Z. Gai, W. Siemons, C. M. Gonzalez, R. Timilsina, A. T. Wong, A. Herklotz, P. C. Snijders, E. Dagotto, and T. Z. Ward, Phys. Rev. Lett. 114, 256801 (2015).
[16] J. R. Petrie, C. Mitra, H. Jeen, W. S. Choi, T. L. Meyer, F. A. Reboredo, J. W. Freeland, G. Eres, and H. N. Lee, Adv. Funct. Mater. 26, 1564 (2016).

[17] W. S. Choi, J. H. Kwon, H. Jeen, J. E. Hamann-Borrero, A. Radi, S. MacKe, R. Sutarto, F. He, G. A. Sawatzky, V. Hinkov, M. Kim, and H. N. Lee, Nano Lett. 12, 4966 (2012).

[18] E. J. Moon, Q. He, S. Ghosh, B. J. Kirby, S. T. Pantelides, A. Y. Borisevich, and S. J. May, Phys. Rev. Lett. 119, 197204 (2017).

[19] Z. Liao, M. Huijben, Z. Zhong, N. Gauquelin, S. Macke, R. J. Green, S. Van Aert, J. Verbeeck, G. Van Tendeloo, K. Held, G. A. Sawatzky, G. Koster, and G. Rijnders, Nat. Mater. 15, 425 (2016).

[20] H. Guo, Z. Wang, S. Dong, S. Ghosh, M. Saghayezhian, L. Chen, Y. Weng, A. Herklotz, T. Z. Ward, R. Jin, S. T. Pantelides, Y. Zhu, J. Zhang, and E. W. Plummer, Proc. Natl. Acad. Sci. 114, E5062 (2017).

[21] L. Yao, S. Inkinen, and S. van Dijken, Nat. Commun. 8, 14544 (2017).

[22] Y. Unutulmazsoy, R. Merkle, I. Rastegar, J. Maier, and J. Mannhart, APL Mater. 5, 042302 (2017).

[23] B. A., J. Jang, Y. Kim, Q. He, R. Mishra, L. Qiao, M. Biegalski, A. Lupini, S. Pantelides, S. Pennycook, and S. Kalinin, ACS Nano 11, 6942 (2017).

[24] H. Jeen, W. S. Choi, M. D. Biegalski, C. M. Folkman, I.-C. Tung, D. D. Fong, J. W. Freeland, D. Shin, H. Ohta, M. F. Chisholm, and H. N. Lee, Nat. Mater. 12, 1057 (2013).

[25] S. J. Callori, S. Hu, J. Bertinshaw, Z. J. Yue, S. Danilkin, X. L. Wang, V. Nagarajan, F. Klose, J. Seidel, and C. Ulrich, Phys. Rev. B 91, 140405(R) (2015).

[26] M. Saghayezhian, S. Kouser, Z. Wang, H. Guo, R. Jin, J. Zhang, Y. Zhu, S. T. Pantelides, and E. W. Plummer, Proc. Natl. Acad. Sci. USA 116, 10309 (2019).

[27] H. Y. Hwang, S.-W. Cheong, P. G. Radaelli, M. Marezio, and B. Batlogg, Phys. Rev. Lett. 75, 914 (1995). 
[28] See Supplemental Material at http://link.aps.org/supplemental/ 10.1103/PhysRevResearch.1.033160 for more details on the growth conditions, XRD and EELS data.

[29] H. Boschker, M. Huijben, A. Vailionis, J. Verbeeck, S. van Aert, M. Luysberg, S. Bals, G. van Tendeloo, E. P. Houwman, G. Koster, D. H. A. Blank, and G. Rijnders, J. Phys. D.: Appl. Phys. 44, 205001 (2011).

[30] T. Gredig, I. N. Krivorotov, P. Eames, and E. D. Dahlberg, Appl. Phys. Lett. 81, 1270 (2002).

[31] H. Fujishiro, T. Fukase, and M. Ikebe, J. Phys. Soc. Jpn. 67, 2582 (1998).

[32] H. S. Kim and H. S. Kwok, Appl. Phys. Lett. 61, 2234 (1992).

[33] H. Béa, M. Bibes, A. Barthélémy, K. Bouzehouane, E. Jacquet, A. Khodan, J. P. Contour, S. Fusil, F. Wyczisk, A. Forget, D. Lebeugle, D. Colson, and M. Viret, Appl. Phys. Lett. 87, 072508 (2005).

[34] J. Dho, N. H. Hur, I. S. Kim, and Y. K. Park, J. Appl. Phys. 94, 7670 (2003).

[35] D. Schumacher, A. Steffen, J. Voigt, J. Schubert, T. Brückel, H. Ambaye, and V. Lauter, Phys. Rev. B 88, 144427 (2013).

[36] C. Ge, K.-J. Jin, L. Gu, L.-C. Peng, Y.-S. Hu, H.-Z. Guo, H.-F. Shi, J.-K. Li, J.-O. Wang, X.-X. Guo, C. Wang, M. He, H.-B. Lu, and G.-Z. Yang, Adv. Mater. Interfaces 2, 1500407 (2015).

[37] Z. T. Xu, K. J. Jin, L. Gu, Y. L. Jin, C. Ge, C. Wang, H. Z. Guo, H.-B. Lu, R. Q. Zhao, and G. Z. Yang, Small 8, 1279 (2012).

[38] M. Medarde, J. Mesot, P. Lacorre, S. Rosenkranz, P. Fischer, and K. Gobrecht, Phys. Rev. B 52, 9248 (1995).
[39] P. G. Radaelli, G. Iannone, M. Marezio, H. Y. Hwang, S. W. Cheong, J. D. Jorgensen, and D. N. Argyriou, Phys. Rev. B 56, 8265 (1997).

[40] E. J. Moon, P. V. Balachandran, B. J. Kirby, D. J. Keavney, R. J. Sichel-Tissot, C. M. Schlepütz, E. Karapetrova, X. M. Cheng, J. M. Rondinelli, and S. J. May, Nano Lett. 14, 2509 (2014).

[41] J. S. Lee, D. A. Arena, T. S. Santos, C. S. Nelson, S. I. Hyun, J. H. Shim, and C. C. Kao, Phys. Rev. B 85, 235125 (2012).

[42] J. A. Mundy, Y. Hikita, T. Hidaka, T. Yajima, T. Higuchi, H. Y. Hwang, D. A. Muller, and L. F. Kourkoutis, Nat. Commun. 5, 3464 (2014).

[43] W. Luo, A. Franceschetti, M. Varela, J. Tao, S. J. Pennycook, and S. T. Pantelides, Phys. Rev. Lett. 99, 036402 (2007).

[44] J. M. D. Coey, M. Viret, and S. von Molnár, Adv. Phys. 48, 167 (1999).

[45] X. Li, I. Lindfors-Vrejoiu, M. Ziese, A. Gloter, and P. A. van Aken, Sci. Rep. 7, 40068 (2017).

[46] A. Tebano, C. Aruta, S. Sanna, P. G. Medaglia, G. Balestrino, A. A. Sidorenko, R. De Renzi, G. Ghiringhelli, L. Braicovich, V. Bisogni, and N. B. Brookes, Phys. Rev. Lett. 100, 137401 (2008).

[47] J. S. Lee, D. A. Arena, P. Yu, C. S. Nelson, R. Fan, C. J. Kinane, S. Langridge, M. D. Rossell, R. Ramesh, and C. C. Kao, Phys. Rev. Lett. 105, 257204 (2010).

[48] K. Takanashi, H. Kurokawa, and H. Fujimori, Appl. Phys. Lett. 63, 1585 (1993). 